\title{
How physics teachers approach innovation: An empirical study for reconstructing the appropriation path in the case of special relativity
}

\author{
Anna De Ambrosis ${ }^{1}$ and Olivia Levrini ${ }^{2}$ \\ ${ }^{1}$ Department of Physics “A. Volta," University of Pavia, Pavia, Italy \\ ${ }^{2}$ Department of Physics, University of Bologna, Bologna, Italy
}

(Received 15 October 2009; published 19 August 2010)

\begin{abstract}
This paper concerns an empirical study carried out with a group of high school physics teachers engaged in the Module on relativity of a Master course on the teaching of modern physics. The study is framed within the general research issue of how to promote innovation in school via teachers' education and how to foster fruitful interactions between research and school practice via the construction of networks of researchers and teachers. In the paper, the problems related to innovation are addressed by focusing on the phase during which teachers analyze an innovative teaching proposal in the perspective of designing their own paths for the class work. The proposal analyzed in this study is Taylor and Wheeler's approach for teaching special relativity. The paper aims to show that the roots of problems known in the research literature about teachers' difficulties in coping with innovative proposals, and usually related to the implementation process, can be found and addressed already when teachers approach the proposal and try to appropriate it. The study is heuristic and has been carried out in order to trace the "appropriation path," followed by the group of teachers, in terms of the main steps and factors triggering the progressive evolution of teachers' attitudes and competences.
\end{abstract}

DOI: 10.1103/PhysRevSTPER.6.020107

\section{INTRODUCTION}

An important reference for science education research in Europe is the so called "Rocard Report," recently published in (2007) [1]. The report illustrates the results of the work of the European Commission aimed at analyzing a selection of the ongoing collaborative science education initiatives within the EU and draws from this analysis a reduced set of concrete policy recommendations. The Report clearly highlights to what extent research in science education has proved to be a relevant source of innovative proposals concerning the choice and organization of teaching or communication contents and methodologies both on science teaching and science teacher education. Nevertheless, it declares also that most of effective innovative proposals have been implemented only in local school contexts. The key players in the renewal of science education and the dissemination of "best practices" are said to be the teachers, and, among other methods, the Report suggests that being part of a network allows them to improve the quality of their teaching and supports their motivation.

The general research issue of this paper addresses some of the Rocard Report recommendations inasmuch as it concerns the problem of how to promote innovation in school via teachers' education and how to foster fruitful interactions between research and school practice via the construction of networks of researchers and teachers.

Research in physics education has produced and is still producing materials explicitly targeted at teachers and devoted to innovate the teaching or learning physics: from the design of teaching learning sequences to long-term proposals (for example, Advancing Physics by Ogborn and Whitehouse [2]).

As research products, innovative materials, also when they refer to specific topics (like Teaching Learning Sequences), are designed to take into account research results
PACS number(s): 01.40.Fk, 01.40.J-, 01.40.ek, 01.40.gb and they are generally based on hypotheses and experiences of the designers, usually different the ones from the others but at the same time potentially correlated in their common aim of substantially changing the traditional teachinglearning process.

Recent researches have however shown how the implementation of research products in the classroom is a problematic task for the teachers.

For example, the STTIS (Science Teacher Training in an Information Society) European Project (1997-2001) [3], aimed at detecting and analyzing transformations between what was expected by implementing a research-based sequence and what is observed when teachers put an innovation into practice, produced very important research results on this issue. In particular it shows that implementation often implies a transformation of the original proposals, sometimes with the loss of important aspects of innovation.

More specifically, many examples of "lethal transformations" [4], distortions and/or specific problems in implementations are known within the research literature. Many of them can be seen as variations of the same tendency among teachers of accepting or refusing the whole proposal on the basis of personal or local criteria and of the same difficulty in moving from a global scale to a local one (and vice versa):

(i) Fragmentation of the holistic view:

"Teachers often restrict themselves to giving a detailed presentation of the topic one step at a time, where it was hoped that they would try to help students grasp the overall scope and nature of the topic. That is, questions about the issues that a given concept addresses, about how the various concepts are linked, and about the overall goal of a given task are not on the agenda" [5].

(ii) Underestimation of the importance of the links between "critical details" and global rationale: "[...] "critical 
details" are not always disregarded by teachers because their grasp of the global rationale is superficial. It may result from a lack of training as to a connection between details and global rationale" [6].

(iii) Modulation of the cognitive complexity of the tasks offered to students:

"Oversimplifying tasks thus could be an important (although not necessarily lethal) mutation for many Learning Sciences-based approaches; it could reduce the students' opportunities for rich cognitive engagement" [4].

As Pierson points out, the alternative local or global in teachers' vision of a proposal is a crucial point since it can influence the feature that mainly distinguishes good teachers: the "prospectiveness" of their actions. "[Good teachers], instead of an evaluation (e.g., "good" or "that's right") that ends the sequence, use the slot to encourage students to elaborate or extend their thinking. A "prospective" move uses the student response to drive the discussion forward-it anticipates that students are capable of more extended argumentation and deeper thinking" [7].

As well as the tendency of being trapped in a local vision, other common attitudes of teachers toward new approaches have been pointed out:

(i) the tendency of transforming and "tuning" the proposal on their own style to obtain intellectual and professional satisfaction (including mixing new with the old) [6];

(ii) the tendency of accepting the challenge of going deep into the proposal if it represents an answer to disciplinary problems recognized as crucial by the teachers [8].

In this paper, the problems related to innovation are addressed focusing on the phase during which teachers analyze an innovative proposal in the perspective of designing their own teaching paths. The results obtained in the study allow us to argue that problems known in the research literature and usually related to the implementation process can be found already when teachers approach the proposal and try to appropriate it. The paper intends to demonstrate that by focusing on the "appropriation process" [9] it becomes possible to provide arguments to support that, while the first tendency (being trapped in a local vision) represents a real obstacle for innovation, the last two tendencies can be transformed into productive resources, if "properly" (in the suitable moments and ways) activated.

As better specified later on, the term "appropriation" means the process of entering the proposal deeply-and extensively-enough to see the teaching of that topic in a different way. Such a process implies one to be able to identify the basic presuppositions on which the proposal itself has been designed as "educational reconstruction" of the topic [10].

\section{STUDY}

The study regards an analysis of the process the teachers go through in order to appropriate the approach to relativity proposed in the text "Spacetime Physics" by Taylor and Wheeler and to compare it with more traditional ones in the perspective of designing their own path for the class activities at the secondary school level.

The work started almost by chance. We decided to collaborate to a web course on the section about Relativity because we had previous experience on this topic. At the beginning we considered it mostly as a new teaching experience, not a real research opportunity. Teachers' reaction and their active participation was a surprise that solicited us to inquire more systematically on teachers' responses and to analyze the data "naturally" collected during the work.

\section{A. Context}

The context of the study is a Master course organized by the University of Udine (director Marisa Michelini) with the collaboration of researchers working in eight Italian Universities. The course dealt with the teaching of modern physics in high school and special relativity was one of the topics.

The course was organized as a web course based on a site, allowing an easy exchange of materials, a forum and the possibility of collaborative writing of documents. Two workshops were held in Udine with the participations both of the teachers attending the course and the researchers.

The Module on relativity was designed and implemented with the aim of giving the teachers cultural and professional tools to recognize the peculiar features of Taylor and Wheeler's proposal and its being the expression of an interpretation of the theory and of its teaching [11].

Twenty teachers were actively engaged in the activities. Their previous experience on the topic was as following:

(i) one teacher had never studied relativity in university courses and her knowledge relied on high school textbook presentations;

(ii) one teacher had studied relativity in a course of the Postgraduate School aimed at certifying secondary school Physics and Maths\&Physics teachers;

(iii) the others studied relativity as part of introductory physics courses or of more advanced courses on theoretical physics (among those teachers, seven of them attended also a specific course on relativity in their university studies).

To favor the appropriation of Taylor and Wheeler's proposal, teachers were guided to compare it with Resnick's proposal [12], up until now the main reference for school teachers and textbook authors [13], and with the results of physics education research on relativity [14].

\section{B. Different approaches to special relativity}

Since the 1960s two different approaches to the teaching of special relativity have been available as prototypes for teachers: Resnick's and Taylor and Wheeler's approaches.

They had different diffusion, at least in Italy, with Resnick's approach more popular in the university courses and in the textbooks for high school students. Taylor and Wheeler's approach is followed only by an elite group of teachers, even though the 1992 version of Taylor and Wheeler's book was conceived to be used by a wide population of students and teachers.

The two textbooks focus on different concepts (the relativistic effects or the invariant quantities and relations) use 
different languages and reflect different conceptions of the theories before and after special relativity.

Resnick's proposal presents the theory by going over the historical evolution and in coherence with the operational approach ascribed to the original papers of Einstein $[15,16]$. The proposal holds the thesis that relativity is "a theory of measurement" and such a thesis is argued by focusing on Lorentz transformations, by using algebraic representations predominantly and by illustrating the physics meaning of the relativistic effects (relativity of simultaneity, length contraction and time delay) by means of the Einstein's thought experiments. Particular attention is paid on the experimental results that led to-and corroborated - the need of revising the conceptual bases of classical kinematics and dynamics.

The success of Resnick's proposal is probably due, besides to the clearness and coherence of the presentation, to the choice of going over some steps of the historical evolution, usually conceived as an effective teaching strategy for softening the impact of a theory that requires space and time views to be deeply revised [17]. Moreover the operational approach, although criticized by many physicists and philosophers as simplistic form of empiricism, has a great persuasive power because of the image of concreteness that seems to give.

The main criticisms addressed to Resnick's approach concern the limits shown by the algebraic-operational language for paving the way to general relativity and for highlighting the formal four-dimensional structure according to which new relations among the dynamical concepts of mass, energy and momentum must be redefined.

More innovative proposals (like Taylor \& Wheeler's one) rely heavily on a geometrical or Minkowskian formulation of special relativity. This approach, which strongly emphasizes the concepts of event, of inertial reference frames, of spacetime interval, of moment-energy, and of invariance, potentially offers an elegant and conceptually transparent nonhistorical reconstruction of the theory. One of its main advantages is paving the way to contemporary physics, including particle physics and general relativity.

Unlike the traditional approach, the authors make the "puzzling" choice of presenting, from the beginning, special relativity as a theory holding in absence of gravitation and, according to the equivalence principle, the inertial reference frames as free-floating systems [18].

Despite its cultural relevance and conceptual transparency, the proposal has some problems of implementation at the secondary school level. The main sources of problems are the "length" of the outlined path (with respect to the time that can be scheduled for relativity within the whole physics curriculum in high school) and its being so unfamiliar that teachers may find it difficult to compare it with the paths they followed as students or present in textbooks. As a result, the approach tends to be oversimplified when implemented. Such oversimplification can lead to the lose of the innovative aspects characterizing the whole proposal and, even worse, to create short circuits that prevent students from overcoming difficulties well-known in literature [19].

\section{Methods and data sources}

The work has been developed with the teachers along three phases:
Phase 1. Analysis of "Space Time Physics" by Taylor and Wheeler in the 1994 Italian version, in order to reconsider basic knowledge about special relativity from an educational perspective and to study the peculiar aspects of an "innovative" approach.

Phase 2. Analysis of Einstein's paper "On the Electrodynamics of Moving Bodies" and of Minkowski's "Space and time," as historical-philosophical roots of, respectively, the approach by Resnick and that by Taylor and Wheeler, in order to reflect on the epistemological implications of different teaching perspectives.

Phase 3. Reflection, carried out by teachers, organized in groups, on problems stemmed by previous work and identification of criteria for designing teaching proposals [20].

In each Phase the work was organized in a similar way:

(i) Analysis of materials carried out by the teachers on the basis of grids prepared by us. Materials examined were the chapters of Taylor and Wheeler's book, Einstein's and Minkowski's original papers, as well as documents commenting the original papers, and research articles about teaching or learning special relativity [21];

(ii) Production by each teacher of a document reporting the results of the analysis in terms of questions they wanted to share in the forum;

(iii) Production by two volunteer teachers of a report where the questions were collected, reorganized and, in case, selected;

(iv) Discussion on a forum triggered by the questions collected in the report.

After Phase 1 and Phase 2 were completed, the teachers filled in a questionnaire aimed at collecting their reflections on the activities carried out and at orienting the following work.

Teachers' initial reaction to the analysis of Spacetime Physics was so lively and multifaceted that it appeared evident, since the beginning, that the appropriation of the proposal would have required the teachers to collectively manage a complex, long, demanding as well as stimulating process. Given the reaction, we decided to observe the phenomenon from a research perspective. At the beginning the research questions were essentially practical: how could the teacher's approach to innovation be interpreted in the light of research results known in literature? How might we, as instructors, manage the process as best as possible?

The research questions progressively evolved toward more and more theoretical ones: How the whole teachers' process of appropriation of an innovative proposal can be modeled (reconstructed)? What general elements could be pointed out from such a rather specific context in order to explain how and why appropriation is hindered or favored by specific features of the training environment?

The considerable amount of qualitative data used for the analysis come from

(i) teachers' reports containing the analysis of the material proposed to them;

(ii) written messages in the forum discussions;

(iii) questionnaires;

(iv) documents produced by the teachers during Phase 3.

The data have been analyzed through a qualitative [22], "phenomenological" [23] methodology. 
According to the research questions and the chosen methodology, the reconstruction of the "appropriation path" has been carried out at two levels: a mainly descriptive one, aimed at modeling the process in terms of main steps followed by the teachers (what happened); a more interpretative one, aimed at pointing out the factors that triggered the progressive evolution of teachers' attitudes and competence (what made it happen; why and how it happened).

\section{RECONSTRUCTION OF THE APPROPRIATION PATH}

For modeling the appropriation path, three steps have been identified:

(i) Accepting the game, i.e., constructing a common way of looking at the problem;

(ii) Playing a common game, i.e., constructing criteria to go deep into Taylor and Wheeler's proposal;

(iii) Exploring the off stage of the game, i.e., how to find the appropriate ground to compare the innovative approach chosen with others.

In the following sections the three steps are described.

\section{A. Accepting the game}

This first step was accomplished approximately during the analysis of chapter 1 and 2 of Spacetime Physics. A shift was observed in teachers' attitudes: Teachers passed from an initial tendency to consider the proposal very locally, to compare it step by step with one's personal experience (as teachers and as students), and to impatiently propose new solutions, up to the consciousness of key problems in the content structure, problems that do not admit local and immediate solutions.

Let us look at examples of the "local" and "reluctant" initial attitude:

"The first question I asked myself when I finished to reading Chapter 1 was about the starting point: in a normal class it is better to start from a real problem, for example from M\&M [Michelson \& Morley] experiment, otherwise I think that a lot of confusion can be generated, in particular among the less motivated students..." (Daniele) [24]

"It would be interesting to develop "real" teaching sequences, single modules where durations, age of students, and other details are clarified because these things limit greatly what we can do..." (Federica)

The teachers' initial impact with the proposal, characterized by a widespread resistance to be personally engaged in a long range reflection, appears as a general tendency to use very local evaluation criteria and the willingness of finding immediate alternatives to Taylor and Wheeler's approach.

At first the most important mentioned criteria were students and personal experience. The reference to presumed student difficulties was for the teachers the greatest point to distinguish "what works and what does not work."

"I have another problem, i.e., the student's age and their confidence with Physics. I often prefer to intro- duce relativity in the last year after electromagnetism, not only because I was interested to follow an historical path, but also because we are talking of students who are approaching physics the first time, its method and its way of investigating nature. In my opinion it is dangerous to introduce Special relativity too early, after the study of classical mechanics, specially if one wants to connect it to General Relativity as T\&W [Taylor and Wheeler] do." (Ada)

Another shared concern was the effectiveness or necessity of an historical approach that sounded more familiar to them:

"I can appreciate T\&W's geometrical approach, but I would prefer to start with an historical introduction to focus on the problems and the experiments that led to the construction of the theory..." (Nadia)

In this step a sensible change occurred in the ways in which teachers refer to students and their personal experience as criteria for evaluating a proposal.

At the end of the analysis and discussion on the second chapter, indeed, the problem of students' understanding or nonunderstanding assumed another meaning: It became the motivation to reflect on the content structure and to reconsider it from a new perspective, where attention is paid more to a global, cultural students' development than to the local acquisition of single concepts.

"How to propose Modern Physics to students? Which experiments, real or mental, which paradoxes, mathematical argumentations, stories, is it convenient to choose to help students understand and construct a reasonable idea of Physics and of its methods?" (Francesco)

A number of statements show how students' needs are now for the teachers a source of questions that solicit reflection and no more a reason for cutting discussions. On the other hand, if initially their own personal experience was the main reference to decide that the history could have a softening and motivating role, while Michelson and Morley's experiment could be a promising beginning, the discussion on chapter 2 produced a new way of looking at one's personal experience: this appeared now problematic and limited, with local incoherence and discontinuity. Teachers realized that a new perspective from which to look at relativity and its role within the whole discipline was necessary for fixing the specific problems come out.

"Someone (I do not remember who) posed the following problem: if we have always taught the students that in classical physics (see Amaldi [25]) an accelerated system is not inertial, how can we make them understand that a free-falling elevator is equivalent to an inertial reference? If I define this room an inertial reference, how can I tell the boys that the falling elevator is an inertial frame too? Of course it is obvious for a space ship far from any gravitational interaction. The elevator feels the gravitational attraction, then (the students would say) why doesn't a little sphere released in it fall? It is difficult to explain our ideas in this context, but I hope that someone understand what I mean" (Ada). 
"I definitely need to clarify to myself the problem of the Newtonian approach (first law and inertial systems) and free floating inertial systems. I did not well understand and I do not want to drop the problem without having compared our opinions. I come to the question: how are the two approaches related? Is Newton however a premise of the discourse or does the "freefalling system" approach allow somehow such a setting up to be, I don't say replaced, but overcome?" (Mario).

Discussion shows how the analysis of the two first chapters of Spacetime Physics led the teachers to positively evaluate the increase of complexity of the thematic area, due to the appearance of new puzzling elements (in particular the new definition of inertial frames) and to consider this as a chance, rather than an obstacle, for their own and students' understanding.

A further point of discussion concerned the narrative style used in the first two chapters (for example in the story "A trip from the Earth to the Moon" that introduces the problem of the free-floating frames): the perplexity of some teachers toward the use of analogies and tales, considered as too light and not rigorous enough, clashed mainly with the strong engagement of socially committed teachers who were looking for renewed languages and styles to communicate an image of physics as social construction.

"In short, through the parable many messages are conveyed to students [she mentions, for example, "the introduction of the invariant as solution or negotiation of the conflict between different ideas and methods, without making one idea prevail on the other"] which contribute to construct an idea of science as CONSTRUCTION or INTERPRETATION. For me it is very interesting!" (Paola)

From a more interpretative point of view, four factors have been singled out for explaining why and how the shift occurred, from the initial distrust to the willingness to analyze the text in more detail. Two of them $(a, b)$ regard some characteristics of the approach and two $(\mathrm{c}, \mathrm{d})$ some peculiarities of the group dynamics favored by the web or by specific mediation strategies. More specifically, they are:

(a) The early presentation of the crucial physical concepts on which the educational reconstruction is based (invariance of the space-time interval, free-falling inertial frame, the problem of the continuity with general relativity, the problem of the clock synchronization in a latticework of clocks). This element represented initially a cause of distrust because of the teachers' tendency, known in literature, of tuning the proposal to their own experience. But it turned to be a productive resource as soon as the teachers recognized weaknesses and inconsistencies in their disciplinary knowledge that could not be solved by local interventions, but that required a wider reconstruction or even a change of perspective. In coherence with other researches on teacher education [8], local elements belonging to the plan of teachers' disciplinary content knowledge proved to be crucial for triggering a more general change in the teaching perspective. They seemed to play the role of "critical details" [6]. (b) The use, in the text, of new communication strategies (like analogies and tales) for introducing new ways of looking at known phenomena. This point acted on some teachers as intuitive and emotion-laden factor for recognizing the proposal as original and innovative. The presence of socially committed teachers provided a new key that allowed the sceptical teachers to reconsider the role of the tales from a more sounded cultural perspective.

(c) The presence of different and complementary voices in the discussion, corresponding to at least three different roles played by the teachers in the group: The free and external observer (free from specific studies on relativity and of previous experiences in teaching it at school); the supporter of new and original positions; the experienced and skeptical observer always trying to anchor the reflection to known class situations. The natural "role game" stemmed from the discussion not only stimulated the reflection but allowed also the creation of nonequilibrium situations with explicit autoregulative mechanisms producing both a personal and a collective growth.

(d) The mediation strategies we enacted for triggering and supporting the discussion. We chose to limit our intervention just to give pace to the discussion and to separate problems of immediate solution from others requiring a long-term reflection and new contributions from the analysis. In particular an important role was played by: the grids of analysis prepared for each chapter; the threads for discussion we proposed in the forum as a synthesis of the main problems identified in our analysis of the teachers' reports; the documents we elaborated after the discussions on the forum for recording the problems remained unsettled. These tools, indeed, appeared to be effective not only for establishing shared points on which tuning the different positions, but also for outlining a frame able to time the various topics of discussion by distinguishing short-, medium- and long-term problems as well as problems lying on different plans (conceptual, curricular, philosophical or didactical).

Just to give an example, after the lively and messy forum on Chapter 1-where Taylor and Wheeler give an overview of the entire proposal-we elaborated a document where the main problems and conflicting points arisen in the discussion were reported. Instead of giving in to the temptation of providing quick - and necessarily partial-answers, we represented the agenda of the whole Module, indicating where the questions would have found, in our opinion, their "natural" moment for discussion. In more detail, we proposed an agenda where we split: (i) problems concerning conceptual difficulties well known in the research literature (to be discussed in short-medium terms during the scheduled activity devoted to the analysis of specific research articles); (ii) perplexities concerning basic choices of the proposal (to be discussed in medium-long term and, in particular, at the end of Phase 1 and during Phase 2); (iii) problems concerning the implementation in class (to be discussed in long term and, in particular, during Phase 3). We made this choice mainly as surviving strategy in front of the avalanche of questions to which we could not practically answer because of the time and ways imposed by the web communication. A posteriori it revealed a choice that changed the general attitude of the teachers' group and of ourselves: the web-communication 
and the necessity of communicating through written texts allowed all of us to keep track of the problems and of the whole process. This awareness contributed to overcoming the attitude of "all-now-fast-and-easy" and to create a more relaxed atmosphere where also different learning rhythms could have room. Moreover it contributed to give the first and strong signal that an appropriation process is a long, complex and multidimensional process.

The main results of the first step can be summarized by saying that, in approaching an innovative proposal, teachers have to first evaluate their reliance on it.

The group of teachers arrived at sharing the conviction that to examine in depth the proposal was worthwhile, since:

(i) the mix between new and old [6] pointed out disciplinary problems, intellectually stimulating, that did not admit trivial and local solutions;

(ii) the confidence of finding, along the whole course, both the time and the ways to develop the many problems stemmed from the first analysis produced a shared willingness of a medium-long term effort.

\section{B. Game and its rules}

The second step (that required about five weeks) was accomplished while the analysis of Space Time Physics was being carried out and completed, and it is characterized by teachers' efforts to find parameters to "measure" the proposal as a whole.

The idea of a student as an ideal unit to measure the validity of a proposal was abandoned together with other generic criteria such as the level of abstraction of the proposal compared with the traditional ones. Teachers discussed what is concrete and what is abstract starting from a comparison of real and thought experiments and found a sensible disagreement in their positions. They wondered what kind of abstraction is necessary to understand physics and what are useless complications that discourage students.

"We do not agree completely; [two of us] think that thought experiments are generally more difficult to understand than the "real" ones because they require not only to develop an abstract logical-deductive reasoning (usually), but also to see the contradictions that the reasoning produces., [On the contrary another one] thinks that thought experiments are understandable just like the real ones. However it is necessary to stress that even real experiments in SR can be only described and not "personally" carried out in the laboratory. At the end they have to be accepted as an act of faith." (Nadia, Andrea, Barbara)

"I agree $[\ldots]$, it is hard for students to understand "thought experiments" both because they are not used to go deep in the meaning of the text, and because some of them lack abstract logical-deductive skills. I am convinced of the educational value of the real experiments and I think that if the students are familiar with real experiments from the very beginning of the physics studies, it is possible, perhaps, to let them reflect on thought experiments." (Simonetta)
"As far as abstraction is concerned, I think that for topics such as relativity, it is not clear if thought experiments are more difficult to understand than experiments on flying $\mu$-mesons. However, the educational value of the two [kinds of experiments] are different: I personally think that students should be educated to mental reasoning so that they can imagine and visualize situations that can help them to construct meanings." (Michela)

The discussion led them to confront the complexity of this issue (with the surprise of some teachers) and the uselessness of the opposition concrete or abstract as unproblematic criterion to judge the quality of different proposals.

Meanwhile another criterion was stemming from teachers' debate: the coherence of a proposal. In particular, teachers' discussion focused on the following questions:

What does it mean to design and to implement a coherent teaching sequence? What are the characteristics of such proposals? What is the reference respect to which one can evaluate its coherence?

These questions are the result of a debate which showed the difficulty of finding a match between the contents of a traditional physics curriculum and an innovative proposal. From this debate the necessity of a coherent path, designed as such since the beginning, was put forward and a reflection on what coherence means was started.

"I think that the main difficulty a student would find in T\&W's approach to special relativity is that many, many concepts they have already learned before are now presented in a radically different way; the student has to make a big effort in order to re-adjust and reorganize their knowledge in the light of the new approach; we can not expect that such a revolution be accepted without difficulties and resistances. How to cope with this problem? Perhaps by getting the students used to looking at the things by different perspectives, to reasoning without rigid framework, to revise their knowledge according to alternative reading keys. Of course this objective requires much time and energy, but it could be worthwhile to try." (Simonetta)

"The problem of coherence would disappear if we redefine all the physics concepts, since the first one, according to T\&W's perspective. It would be necessary to define the inertial frame as a free floating frame $[. .$. since when we introduce the Newton's laws [...]. But what is the price?" (Nadia, Andrea, Barbara)

"This is my idea: in the first three chapters T\&W seem to set the stage for a future "squaring of the circle." In chapter 4 they "open the discourse" by telling the story of the trip to Canopus so that one can see some consequences of what was previously introduced. Then problems of coherence are in the initial construction phase. They can be solved only at the end of the whole path." (Mario)

Different positions were expressed by the teachers about the coherence of a sequence:

(i) coherence as logical development of a path from clas- 
sical to modern physics, as a result of a radical reconstruction process of the physics contents. (This does not mean that gaps and differences are not dramatically highlighted as for example in the Parable of the Surveyors at the beginning of Taylor and Wheeler's book);

(ii) coherence as historical development of a path, where conceptual discontinuity between the different theories historically developed are underlined;

(iii) coherence as systematic use of the experimental method characteristic of physics inquiry

["I think that the problem of coherence is a fundamental one, but at the same time it is insoluble...Hence I think that a real coherence can be found only in the "EXPERIMENTAL METHOD," the pivot around which different descriptions can rotate, all COHERENT with the method itself." (Matteo)]

(iv) coherence as reconstruction of the physics contents starting from fundamental concepts and categories such as the space-time description, causality, determinism.

A shared opinion stemmed from this debate:

"In order to grasp the coherence of an approach it is necessary to internalize the meaning of a theory far beyond its formal aspects. It implies to go deep, to analyze its implications, to acquire different perspectives and interpretations. It means to know both the origin of the hypotheses and their consequences. The task is not easy for a teacher." (Michela)

The discussion about coherence has been crucial both for the teachers and for us:

(i) for the teachers, the discovery of the plurality of perspectives among them opened new interest for investigating the specific kind of coherence that could characterize the unusual Taylor and Wheeler's proposal [26];

(ii) for us, the discussion was a clear signal that the initial attitude of distrust and resistance was changed, since they were no longer stuck on very small local aspects, but they were ready for a medium-term, patient and resolute, analysis up to the critical point where it was possible not only to discover the details, but also to attain a global perspective on the proposal.

The main factor supporting the process has been the lively dynamics that allowed the maturation of a shared conviction that global evaluation criteria are needed for justifying local choices.

Other crucial factors have been:

(i) the growing awareness that physical problems, specifically addressed by this proposal, were not solved clearly in a traditional approach. This is the case of the idea of inertial frame, of the new relation connecting momentum, energy and mass as well as the perspective view on general relativity. About the concept of mass, for example, Vincenzo proposed for the collective discussion the following point:

"I would like to discuss the concept of "relativistic mass." It is true, often in the textbooks the "relativistic mass" is introduced as $m_{r e l}=m \gamma$. It depends, by definition, on the velocity and one pretends to give it an independent meaning. [...] But its interpretation is an- other problem. It is enlightening what Whitaker writes in his paper [27]: "The most important criticism of the relativistic mass approach is that it gives the impression that the effects of relativity are due to 'something happening' to the particle, whereas they are of course due to the properties of space-time." (Vincenzo)

(ii) The acknowledgment of specific problems of continuity or discontinuity with classical physics, that progressively made the teachers more and more willing to rethink about teaching or learning classical physics:

"Usually one thinks that using the classical or the relativistic mechanics is a matter of velocity of the bodies involved. Don't you think that these two chapters well emphasize that the relativistic theory not only describes the high speed boundary cases, but also gives explanations not achievable with classical mechanics? Take for example the rest energy of a body. It is not correct to affirm, as some textbooks-particularly the eldest ones-do, that the relativistic theory includes the classical mechanics without falsifying it." (Vincenzo)

At the end of the work, teachers agreed on the reliance and relevance of the proposal as the following comments show:

"I was shocked by T\&W's ability in dealing with Relativity, free themselves from traditional approaches, in particular the historical one..." (Pietro)

"One of the most important contributions of this approach is to revise the theory in a coherent and well structured frame so that it does not reduce to the extravagance of the relativistic effects" (Lucia).

"The book gives a geometrical vision of the special relativity and this vision allowed me to "perceive" the possible lines of development of the general relativity. I think it is very important because I was solicited to continue the study toward general relativity, as a necessary development of the special relativity (which otherwise can be trivialized)." (Matteo)

Nevertheless the teachers expressed the need to go deep into specific topics, particularly the ones where a discontinuity was perceived between the new approach and more traditional ones.

They also pointed out that Taylor and Wheeler's proposal is based on implicit hypothesis and views (didactic and epistemological) and that, if preliminary assumptions are not clarified, the comparison with other proposals becomes difficult and the teachers are forced to follow a unique reasoning strand.

"The proposal appears somehow as unilateral. [...] I feel however a kind of mistrust (perhaps only intuitive) toward the question. As we were in front of a sort of situation forced by [the need of] searching for an excessive intelligibility in the relations between the physical quantities" (Mario).

"I would like to focus on the hypotheses at the basis of 
T\&W's approach: I feel that there are non explicit hypotheses or that I got lost along the path... I would like to improve my understanding of the whole proposal and be able to compare it with others around" (Anna).

The teachers' debate shows, hence, the following crucial point: Understanding the content is not enough to grasp the general meaning of a proposal and to feel comfortable with it; teachers manifest the need to have tools for disassembling and reassembling it, for going deep into its epistemological and cognitive assumptions (to what image of physics and physics teaching it is related), for comparing it with others (especially the typical textbook ones), and for adapting it to one's teaching or learning attitudes and constraints. These problems were tackled in Phases 2 and 3 of the Module, which were carried out in parallel.

\section{Exploring the off stage}

The problem of how to foster the appropriation process of Taylor and Wheeler's approach became, at this point, how to answer to the teachers' requirements about:

(i) deepening the study of particular aspects of the content matter, in particular the idea of inertial reference frame and relativistic dynamics;

(ii) investigating the basic assumptions of the approach so that to make it comparable to more familiar approaches.

The first requirement was addressed in Phase 3 where teachers, by working in groups, reflected on the topics previously identified as crucial and started to sketch possible teaching paths, as a kind of "design exercise" [28].

The second requirement triggered the third step of the appropriation process, during which the "off stage" of Taylor and Wheeler's proposal has been analyzed and the perceived distance between this proposal and the more familiar approach by Resnick has been interpreted.

With the help of materials prepared by one of the authors (OL) and by other researchers of the Bologna group [29], teachers examined passages from Newton's Principia and the criticism to them by Leibniz and Mach. Then the work has been focused on the analysis of the original memories of Einstein ("On the Electrodynamics of Moving Bodies," 1905) and of Minkowski ("Space and time," 1908), carried out in order to find the historical roots of the main teaching proposals. The leading thread of the analysis has been the debate on the concepts of space and time in Physics, and the different interpretations of relativity proposed by Einstein and Minkowski have been reread as contributions to such a debate [30].

The aim of this phase was to show the coexistence of different representations of the theory at the beginning, but the development of just one of them (Resnick's one) for teaching purposes (starting from university teaching).

The discussion with the teachers, based on the materials given to them, pointed out to what extent the choice of focusing, in the educational tradition, on only one approach strongly influenced the proposal itself: The choice indeed triggered a sterilization process that progressively, from one generation of students to another, led to forget the basic cul- tural features of the proposal and its specificities with respect to other possible approaches.

The progressive loss of the interpretative dimension produced a sterilized dominant approach (in this case grounded on Resnick's proposal) and the implicit formulation of criteria for evaluating the other approaches in terms of "distance from" the proposal assumed, often out of habit or by trust, as the "best" (or the most "natural") [31].

"I think that "sterilization" is not only a limitation, but an epistemological choice: It is the consequence of the idea that Physics must be unproblematic, to have no different points of view, different images of the world. This seems possible only for the research frontier, but for the other questions just one world image can be handed down... This is also happening for relativity since it is already more than 100 year old." (Carlo).

One of the key factors that led the teachers' group to see as worthwhile a critical reflection on the process that made one approach become dominant over the other has been the acknowledgment of the effectiveness of Taylor and Wheeler's approach in favoring a deep understanding, of its inner global coherence as well as of its potential in paving the way to understanding Wheeler's geometrodynamic interpretation of general relativity.

The second factor triggering the process has been the analysis of the off stage of the two reference proposals, i.e., the reconstruction of their cultural bases. This analysis allowed the teachers to put the proposals on the same ground and to compare them as different choices of content reconstruction, inspired by different global views of the theory and of its teaching, thus overcoming the tendency of evaluating them as "orthodox or heterodox" alternatives.

This awareness guided teachers to critically review not only textbook proposals, but also their own teaching ways, by removing the absolute value they often assume.

The overall outcome of the work carried out during Phases 1 and 2 is summarized by a piece of evidence that struck us and one teacher stressed in the final questionnaire:

"I was surprised by the "harmony" of the discussion on epistemological issues. Generally these discussions reveal very rigid points of view and irreducible convictions..." (Mario).

As well as the high quality of the written tasks and discussions, indeed, the tone used during the opinions' exchange was evidence of something interesting that was happening to the group as a whole, since they naturally confronted one other in a passionate way, without arriving at irreducible positions.

"I think that the communication system contributed to the harmony: When you write you have to reflect, to revise, to adjust, while if you speak "verba volant'" (Francesca).

But we think that the tone of the discussion was due also-or mainly - to the growing awareness of the complexity of the problems one has to overcome in order to innovate the teaching of modern physics:

"Confrontation with the others has been often very 
useful for clarifying one's own position, or for stimulating a personal reflection on a new issue. Perhaps the very quiet tone depends on the complexity of the problems on which it is difficult to have a conclusive opinion" (Pietro).

At the end of the whole work, many teachers felt the need of expressing their satisfaction by revealing a strong personal involvement stimulated by the discussions occurred. The complexity of the cognitive, cultural, and social dynamics triggered by the multidimensional analysis of a physical topic, as well as the confrontation with open-ended questions, became a new perspective for them from which to look also at the motivational problems they have to address (as they said) in their teaching.

"If one had here presented physics to us as "something ready," it would not have had the same flower. Well, there is an effort, research and study behind this discipline, there are paths taken up and never finished, others dropped because of "prejudices," why ignore all of this and not let them be part of teaching?" (Anna)

"In my opinion the depersonalization of scientific knowledge is a "choice" consolidated, by now, within Physics teaching. I was very glad to reflect on these issues, since it allowed me to think about teaching from a perspective almost never used. I would answer in such a way: according to our way of approaching the discipline (also by considering the previous teaching) we are pretty sure of making physics comprehensible to students; nevertheless we have the big problem of creating motivations and "passion" for the discipline: from this point of view, perhaps, a more "personalized" approach can help us" (Lucia).

\section{CONCLUSIONS}

This paper concerns a study carried out on data "naturally" collected within an online teacher training Master course. The data seemed to be particular suitable to document the evident evolution of teachers' attitude toward an innovative teaching proposal: From an initial hesitation and distrust toward single aspects of the proposal, in conflict with the traditional approach, to the acceptance of the challenge of going deep into the analysis to understand the origins and the meaning of the differences with respect to other approaches.

Because of such evidence we decided to carry out a qualitative analysis of data aimed at reconstructing that collective process we called "appropriation path." The reconstruction has been developed at a descriptive and interpretative level, respectively in terms of steps followed by the teachers (what happened) and of factors that triggered the progressive evolution of teachers' attitudes and competencies (what made it happen; why and how it happened).

While the three steps individuated (accepting the game after an explicit or implicit evaluation of its authoritativeness; playing the game by tuning local and global aspects; exploring the off-stage of the game, by comparing the novelties with previous knowledge and experience) are quite general and can be maybe related to every process of appropriation, the factors triggering the process are at a different level of specificity. In particular, the study allowed us to find out:

(i) what made the proposal authoritative for these teachers (for example, the early introduction of disciplinary crucial problems that could not be solved by local changes in a traditional approach or the use of tales and analogies);

(ii) what global criteria teachers collectively discussed and problematized also for a local evaluation of the proposal (for example, the discussion on coherence and on the dichotomy abstract or concrete or the "comparability" of the proposal with more traditional ones);

(iii) what supported the evolution of teachers' attitude (for example, the role game in the group dynamics and specific mediation strategies).

The study can be framed in the big issue of how to foster innovation via teacher education, and our findings fit in with (can be triangulated with) the ones known in literature and usually related to the implementation of innovation. We indeed observed the well-known tendency of transforming and tuning the proposal on one's own style to obtain intellectual and professional satisfaction [6], as well as the tendency of accepting the challenge of going deep into the proposal if it represents an answer to disciplinary problems recognized as crucial by the teachers [8]. But also, the positive potential of these tendencies in triggering the appropriation process appeared very clearly from the data, as described in Sec. III A, particularly in points (a) and (c).

As already mentioned in the introduction, the notion of "critical details" has been defined, by Viennot, so as to stress the difficulties met by teachers in tuning the global rationale of a proposal and its local features. It is also argued that teachers' disregarding of critical details may be the consequence of not suitable training [6]. The experience reported here is not only an example of how the local-global issue has been collectively and positively faced during training activities, but it also provides advancement in the analysis of what can foster or hinder the move for attaining a global perspective. The dynamics of the discussions about "abstract vs concrete" and "coherence," for example, shows how widespread is the belief that univocal and unquestionable ways do exist for defining general criteria like those. The collective discovery of their problematic nature has been, in our experience, a fundamental moment for putting the teachers in the mood for investigating new and unexpected possibilities of building an unusual global rationale and of reconsidering the details according to new global lenses.

Moreover, the study showed that appropriation is not only a game of tuning global and local aspects but also a game of making a proposal "comparable" with others. This finding represents a real challenge not only for teachers, but also for teacher trainers and researchers. The process of making explicit the elements needed for making innovative proposals comparable with the more familiar ones requires indeed consistently manage a proposal at different levels (details, rationale, behind presuppositions), coordinate different dimensions of knowledge (disciplinary, cognitive, philosophical and educational) and, at the same time, interpret and exploit the different styles and needs of the people involved in the 
community of work. For addressing such a complexity, it has been fundamental for us to manage the whole module of relativity in collaboration, as well as to share some complementary competences we had: The expertise of the first author (ADA) in Taylor \& Wheeler approach and the experiences matured by the second author (OL) in exploiting historical materials for teaching.

To conclude, we mention two specific research issues that remain unsolved and that could provide new relevant contributions to the analysis presented here:

(i) what personal trajectories [32] teachers followed within the collective discussion and what possible connections between individual and collective growth can be pointed out;

(ii) how the appropriation process affects the implementation of the proposal in classroom or, more in general, how the description or interpretation of the appropriation process we have obtained can be checked in its reliability.

The first questions are part of our current research agenda as further level of analysis of the data already available. The second ones deserve, instead, the design of further experiments and new data collection.

\section{ACKNOWLEDGMENTS}

The authors wish to thank M. Gagliardi, N. Grimellini Tomasini, and P. Guidoni, as readers of previous drafts of the paper for their comments and suggestions that improved the content and presentation. Manuscript reviewers for the journal helped refine and clarify arguments.
[1] European Commission, Science Education Now: A Renewed Pedagogy for the Future of Europe (Office for Official Publications of the European Communities, Luxembourg, 2007) (http://ec.europa.eu/research/science-society/ index.cfm?fuseaction $=$ public.topic\&id $=1100$ )

[2] Advancing Physics AS, edited by J. Ogborn and M. Whitehouse (Institute of Physics Publishing, Bristol, Philadelphia, 2000); Advancing Physics A2, edited by J. Ogborn and M. Whitehouse (Institute of Physics Publishing, Bristol, Philadelphia, 2001).

[3] For more information about the STTIS project, see http:// antalya.uab.es/crecim/websttis/general/index.html

[4] J. Roschelle, N. Shechtman, S. Hegedus, J. Pierson, M. McLeese, and D. Tatar, Cognitive Complexity in Mathematics Teaching and Learning: Emerging Findings in a Large-Scale Experiment, ICLS Conference, Utrecht (2008).

[5] R. Pintò, Introducing curriculum innovations in Science: identifying teachers' transformations and the design of related teacher education, Sci. Educ. 89, 1 (2005).

[6] L. Viennot, F. Chauvet, P. Colin, and G. Rebmann, Designing strategies and tools for teacher taining: The role of critical details, examples in optics, Sci. Educ. 89, 13 (2005).

[7] J. Pierson, The Impact of Moment-to-Moment Discourse Moves on Opportunities to Learn Mathematics, Paper presented at the 29th Annual conference of the North American Chapter of the International Group for the Psychology of Mathematic Education, Lake Tahoe, Nevada (2007).

[8] B. S. Eylon, E. Bagno, Research-design model for professional development of teachers: Designing lessons with physics education research, Phys. Rev. ST Phys. Educ. Res. 2, 020106 (2006).

[9] We are grateful to P. Guidoni for suggesting the term "appropriation" also in connection with Vjgotsky view of cognitive dynamics.

[10] U. Kattmann, R. Duit, H. Gropengießer, and M. Komorek, Educational Reconstruction-Bringing Together Issues of Scientific Clarification and Students' Conceptions, paper presented at the Annual Meeting of National Association of Research in Science Teaching (NARST), St. Louis (MI) (1996).
[11] E. F. Taylor and J. A. Wheeler, Spacetime Physics (Freeman and Company, New York, 1992).

[12] R. Resnick, Introduction to Special Relativity (Wiley, New York, London, 1968).

[13] In Italy two important experiences were developed in the past for improving the teaching of relativity at secondary school level: (i) the Relativity Project, directed by G. Cortini (19731979) which involved a number of teachers and researchers from Italian universities (Cortini G. et al., Iniziativa relatività: "Vedute recenti sull'insegnamento della relatività ristretta ad un livello elementare," Quad. G. Fis., II, n. 4 (1977), 13; and G. Cortini, La relatività ristretta, Loescher, Torino, 1978; (ii) The Fabri's proposal (E. Fabri, Insegnare relatività nel XXI secolo: dal 'navilio' di Galileo all'espansione dell'Universo, Quaderno 16 La Fisica nella Scuola, 2005 (first version published in 1989). In this context we decided to limit the analysis to Taylor and Wheeler's and Resnick's proposals because of their international diffusion and because they have been considered sufficient to pose the problem of teachers' appropriation we wanted to address.

[14] See for example, R. E. Scherr, P. S. Shaffer, and S. Vokos, Student understanding of time in special relativity: Simultaneity and reference frames, Am. J. Phys. 69, S24 (2001); R. E. Scherr, P. S. Shaffer, and P. S. Vokos, The challenge of changing deeply held student beliefs about the relativity of simultaneity, ibid. 70, 1238 (2002); G. J. Posner, K. A. Strike, P. W. Hewson, and W. A. Gerzog, Accommodation of a scientific conception: Toward a theory of conceptual change, Sci. Educ. 66, 211 (1982); A. Villani and J. L. Pacca, Students' spontaneous ideas about the speed of light, Int. J. Sci. Educ. 9, 55 (1987).

[15] P. W. Bridgman, The Logic of Modern Physics (MacMillan, New York, 1927).

[16] P. W. Bridgman, Einstein's theories and the operational point of view, Albert Einstein: Philosopher-Scientist, edited by P. A. Schilpp, Open Court, La Salle, IL, (1949).

[17] G. Holton, Thematic origins of scientific thought, Kepler to Einstein (Harvard University Press, Cambridge, MA; London, England, 1973) (revised version 1988). 
[18] The choice of defining inertial frames as free-floating systems gives a strong cultural imprinting to the whole approach but it is not strictly necessary in the conceptual reconstruction. The whole reasoning indeed remains logically and conceptually coherent also skipping over Chapter 2 ("Floating free"). The evaluation of pros and cons of this specific choice is very provocative for the teachers. It represented indeed one of the point more widely discussed during the Module on relativity we are here analyzing.

[19] O. Levrini and A. A. diSessa, How students learn from multiple contexts and definitions: Proper time as a coordination class, Phys. Rev. ST Phys. Educ. Res. 4, 010107 (2008).

[20] The work of supporting teachers in their class activities was not scheduled as part of the relativity Module managed by us. Small experiments in class, scheduled as last activities in the whole Master, were indeed supervised by the local responsible person, since teachers attending the Master came from many different towns in Italy.

[21] In particular the papers analyzed are the ones reported in Ref. [15], papers mentioned by Taylor \& Wheeler as reference for problems and exercises, papers about the problem of the interpretation of the mass in special relativity for example, C. G. Adler, Does mass really depend on velocity, dad? Am. J. Phys. 55, 739 (1987); M. Whitaker, Definitions of mass in special relativity, Phys. Educ. 11, 55 (1976).

[22] See, for example, V. K. Otero and D. Boyd Harlow, Getting Started in Qualitative Physics Education Research, http:// www.compadre.org/PER/document/ServeFile.cfm?ID $=9122 \&$ DocID $=1218$

[23] The word "phenomenological" is meant to stress that data have been analyzed in order "to derive trends and patterns through multiple reads of the data, instead of applying a priori criteria or assumptions and seeks to determine whether the data are consistent with them" [22]. In many contexts this methodology is called "inductive," in opposition to "deductive." We prefer to use the word "phenomenological," because of its evocative power in focusing more on the central role of phenomenology, than on the logic through which the conclusions are inferred.

[24] The names are invented.
[25] "Amaldi" is the author of a physics secondary textbook, widespread in Italy.

[26] The coherence that characterizes Taylor and Wheeler approach can be recognized by focusing on two big choices that orient both the overall structure of the book and the ways of addressing the details: the choice of considering special relativity in the light of general relativity and the choice of analyzing all the concepts of special relativity from the geometrical perspective.

[27] The teacher is referring to the paper by Whitaker (see note 21), given to the teachers as support for the analysis of the chapters 7 and 8 of the Taylor \& Wheeler text.

[28] The work carried out in Phase 3 represented an important moment of personal consolidation, but data collected through the web facilities are not suitable to reconstruct the trajectory of each teacher.

[29] In particular, the other people involved in the production of the materials used are: N. Grimellini Tomasini, C. Casadio, M. Clementi, P. Fantini.

[30] O. Levrini, The substantivalist view of spacetime proposed by Minkowski and its educational implications, Sci. Educ. 11, 601 (2002).

[31] The process of sterilization is very evident if one compares Einstein's original memories and the Resnick's approach with their educational transposition in the secondary school textbooks. In particular, the interpretative dimension and the epistemological richness present both in Einstein's papers $[15,16]$ and in the Resnick's text [12] disappear in the textbooks were the operational approach becomes a series of statements unquestionable and apparently neutral.

[32] J. Confrey, in The Cambridge Handbook of The Learning Sciences, edited by K. Sawyer, (Cambridge University Press, Cambridge, England, 2006), pp. 135-152; O. Levrini and P. Fantini, B. Pecori, M. Gagliardi, M. T. Scarongella, and G. Tasquier, A Longitudinal Approach to Appropriation of Science Ideas: A Study on Students' Trajectories in Thermodynamics, Proceedings 9th International Conference of the Learning Sciences, "Learning in the Disciplines, June 29-July 2, 2010 Chicago, IL. 